\title{
Health-Related Quality of Life in Tuberculosis
}

\section{Patients in Eritrea: Comparison Among} Drug-Susceptible and Rifampicin/

\section{Multidrug-Resistant Tuberculosis Patients}

\author{
Zenawi Zeramariam Araia (iD) \\ Araia Berhane Mesfin ${ }^{2}$ \\ Amanuel Hadgu Mebrahtu' \\ Adiam Ghebreyohanns Tewelde ${ }^{3}$ \\ Asmerom Tesfagiorgis Tewelde ${ }^{3}$ \\ Solyana Ngusbrhan Kidane ${ }^{4}$ \\ 'National TB and Leprosy Control \\ Program, Communicable Disease \\ Control Division, Ministry of Health, \\ Asmara, Eritrea; ${ }^{2}$ Communicable \\ Diseases Control Division, Ministry of \\ Health, Asmara, Eritrea; ${ }^{3}$ Integrated \\ Disease Surveillance and Response, \\ Ministry of Health, Asmara, Eritrea; \\ ${ }^{4}$ Health Systems, WHO Country Office \\ for Eritrea, Asmara, Eritrea
}

Background: Despite the negative impact of tuberculosis (TB) on patients' quality of life, TB control programs focus on biological and clinical parameters to manage and monitor TB patients. In our setting, patients' perception of their experience with TB and the impacts of TB on patients' physical, mental, and social wellbeing remain unknown.

Objective: The objective of this study was to evaluate the health-related quality of life (HRQOL) among rifampicin/multidrug-resistant TB (RR/MDR-TB) in comparison to drugsusceptible TB (DS-TB) patients in Eritrea.

Methods: A cross-sectional study was conducted in RR/MDR-TB and DS-TB patients under treatment. Anonymized data collected using the WHOQOL-BREF questionnaire were analyzed using SPSS version 23. Frequency, mean and standard deviation were used to describe the data. Mean group score comparison and relationship between variables were assessed using $t$-test. Domain score was calculated with a mean score of items within each domain and scaled positively, a higher (increasing) score denoting a higher quality of life. Internal consistency was measured using Cronbach's alpha and statistical significance was set at $\mathrm{p}<0.05$

Results: A total of 92 patients (46 RR/MDR-TB and 46 DS-TB) participated in the study. Environmental $(40.63 \pm 10.72)$ and physical domains $(61.80 \pm 17.18)$ were the two most affected domains in RR/MDR-TB and DS-TB patients, respectively. The psychological domain was the least affected domain in RR/MDR-TB $(48.28 \pm 20.83)$ and DS-TB patients (76.63 \pm 15.32$)$. RR/MDR-TB patients had statistically lower mean scores in all domains than DS-TB patients.

Conclusion: HRQOL was impaired in both groups, but RR/MDR-TB patients had a worse health-related quality of life.

Keywords: quality of life, multidrug-resistant TB, patient-reported outcome, end TB, subSaharan Africa

\section{Background}

Tuberculosis is a significant public health concern in many countries. In 2019 alone, an estimated 10 million people were sick with TB, and 1.4 million people died. ${ }^{1}$ Among those diagnosed with TB, 465,000 cases were resistant to rifampicin (RR) and at least to both rifampicin and isoniazid (MDR-TB). ${ }^{1}$ This huge disease burden creates a persistent threat to global tuberculosis care, control, and prevention. ${ }^{[1]}$ In
Araia

National TB and Leprosy Control

Program, Communicable Disease Control

Division, Ministry of Health. P.O. Box

2389, Asmara, Eritrea

Tel +291-I-122129

Email zenhopncl@gmail.com 
Eritrea, despite a declining trend in the notification, TB remains one of the leading causes of mortality in adults. ${ }^{2}$ Moreover, RR/MDR-TB is recognized as an emerging threat to the control of tuberculosis with a prevalence of $2.0 \%$ among new and $7.5 \%$ among previously treated TB cases. $^{3}$

Drug susceptible TB patients (DS-TB) are treated with first-line drugs for six months. The drugs in this regimen are less toxic, and all orally administered fixed dose combinations. Whereas RR/MDR-TB is usually treated for 920 months, ${ }^{4}$ and its treatment requires a course of secondline regime that is more toxic and expensive, including prolonged periods of injectable medications. ${ }^{1,5}$ Despite this expensive and lengthy treatment, only $57 \%$ of the RR/MDR-TB cases have been successfully treated as compared to $85 \%$ of the DS-TB cases at the global level, mainly due to high rates of lost to follow-up, death, and treatment failures. ${ }^{1}$ Furthermore, MDR-TB patients experience serious psychosocial problems due to the disease and its treatment complications ${ }^{6}$, which negatively impacts patients' health-related quality of life. ${ }^{7-9}$

The World Health Organization (WHO) defines the quality of life as individuals' perception of their position in life in the context of the culture and value systems in which they live and concerning their goals, expectations, standards, and concerns. ${ }^{10}$ It is a wide-ranging perception affected in a complex way by the person's physical health, psychological state, level of independence, social relationships, personal beliefs and their connection to noticeable features of their environment. ${ }^{10}$ The negative impacts of TB encompass physical, psychological, social, and financial effects. ${ }^{11-13}$ Thus, TB is associated with anxiety and depression symptoms, and as a result of hospitalization, stigma, and challenges in supporting family life, patients develop social isolation and fail to get the social support that is essential to keep healthy status. ${ }^{14}$ Furthermore, the direct impact of RR/MDR-TB on the quality of life extends to patients and their families. ${ }^{11}$ Consequently, TB patients have a more inferior quality of life than healthy individuals ${ }^{15,16}$, and the impairment in healthrelated quality of life (HRQOL) is worse in RR/MDRTB than drug-susceptible TB patients. ${ }^{17-19}$

Though TB treatment improves patients' HROL, ${ }^{7,15,20}$ some anti-TB drugs cause a significant number of devastating adverse effects that include permanent deafness and symptomatic peripheral neuropathies in patients treated for RR/MDR-TB. ${ }^{5,21,22}$ The HRQOL is affected throughout the disease duration and even after treatment completion. $^{8,23,24,}$ These impairments in HRQOL may be partly related to the physical symptoms, residual physiological harm resulting from the diseases and/or its treatment $^{12}$, and social problems that continue to exist even after the person is cured of the disease. ${ }^{6}$

Patient-reported outcomes provide distinctive evidence and experience on different aspects of living with the disease and their importance and values to the patients. This evidence goes beyond the routine clinical and biological parameters, and is in line with the integrated nature of health, comprising physical, mental, and social wellbeing. The assessment of HRQOL using patient-reported outcomes allows for a comprehensive understanding of how health, disease evaluation, and treatment impact individuals' overall health condition, patient care, and daily life. ${ }^{14,24}$ Despite the above-stated impacts of the disease and its treatment on patients' HRQOL, patients' perception of their experience with TB remains undiscovered in our setting. Thus, the objective of this study was to evaluate the health-related quality of life of RR/MDR-TB compared to drug-susceptible TB patients in Eritrea.

\section{Methods \\ Study Design}

This study was a comparative cross-sectional study.

\section{Study Setting and Participants}

This study was conducted at Merhano RR/MDR-TB national referral hospitaland Maekel region TB treatment centers. This study enrolled all RR/MDR-TB patients and an equal number of consecutive DS-TB patients from TB treatment centers in Maekel region for comparison purposes.

Merhano RR/MDR-TB national referral hospital, located 15 kilometers south of Asmara, the capital city of Eritrea, is the only designated site for the management of RR/MDR-TB patients in the country. Upon diagnosis with RR/MDR-TB, all patients are referred and then admitted to Merhano hospital, where initial evaluation, patient registration, and second-line anti-TB treatment are started. The country has recently adopted the short all oral treatment regime as to the WHO recommendation and is expected to start enrolment. Patients are hospitalized for the duration of the intensive phase (6-8 months) and until their two consecutive sputum samples are converted to a negative by culture test. In the continuation phase, patients are provided with second-line anti-TB medicine 
for 1-3 month stock and receive treatment supervision from the nearest health facility, responsible for organizing DS-TB treatment. Patients must attend the national RR/ MDR-TB hospital and respective zonal referral hospital for their monthly follow-up. Additionally, all patients must visit the national RR/MDR-TB hospital at the end of the continuation phase, end of treatment, in case of treatment failure, or in case of development of significant complications or side effects.

On the other hand, all DS-TB patients are treated in an ambulatory care approach in health facilities nearest to their residence and at a community level with the help of community health workers. All follow-up tests and treatment response evaluations are done in their respective treatment-providing facilities. In both DS-TB and RR/ MDR-TB, all TB services (diagnosis, treatment, treatment response monitoring and related admission, health education and counselling) are provided free of any charge. In addition, RR/MDR-TB patients receive monthly pocket money for the duration of the treatment.

Study participants were $\geq 15$ years old and were under treatment (both in intensive and continuation phase) during the study period. Written informed consent was collected from all participants of the study. In case of participants under 18 years of age, parental informed consent was obtained. Moreover, the data were collected anonymously and did not include any personal identifiers to ensure confidentiality.

\section{Instruments and Data Collection}

Data were collected by researchers during face-to-face interviews using the WHOQOL-BREF scale questionnaire. WHOQOL-BREF is a short version of the WHOQOL-100, a generic instrument to measure the subjective quality of life. This standard questionnaire consists of 24 questions to assess individuals' perception of quality of life in four domains, including physical health, psychological, social relationships, and environment, and two items on the overall quality of life and general health. ${ }^{25,26}$

All questions were followed by local language translation in parenthesis. The translation was made with the help of an expert and piloted before actual data collection. During data collection, the questions were read out to participants in a local language. The WHOQOL-BREF questionnaire has no cut off points that can be defined as good or poor quality of life. Hence, the study did not use any cut off points to dichotomize cases as having good or poor quality of life.

\section{Data Analysis}

Anonymized data were entered and analyzed using IBM SPSS version 23. Descriptive statistics like frequencies, mean and standard deviation were used to describe the data. T-test was used to assess the mean group score comparison of both groups. The mean score of items within each domain was used to calculate the domain score. The four domain scores indicate an individual's perception of quality of life in the respective domain. Each item was scored from 1 to 5 on a Likert scale. Then, raw scores were converted into a linear scale from 0 to 100 , a higher score indicating a better quality of life. $^{25,26}$ In this study, $\mathrm{p}<0.05$ was considered as statistically significant, and internal consistency was measured using Cronbach's alpha.

\section{Results \\ Profile of Study Cases}

A total of 92 TB patients (46 RR/MDR-TB and 46 drug susceptible TB) participated in the study. Generally, males were dominant, 59 (64.1\%) accounting to $33(71.8 \%)$ of the RR/MDR-TB and 26 (56.52\%) of drug susceptible TB patients. According to age distribution, 26 (56.5\%) of DSTB, $32(69.6 \%)$ of RR/MDR-TB and overall 58 (63\%) of all cases were below 45 years old. Proportionally more than half $48(52.2 \%)$ of all study participants, $27(58.7 \%)$ of the RR/MDR-TB and 21 (47.5\%) of drug susceptible TB patients were married/living as married. Among all study cases, $73(79.9 \%)$ were educated, with the highest proportion $39(84.8 \%)$ of school attendance observed among drug susceptible TB patients as compared to 34 (73.9\%) among RR/MDR-TB patients (Table 1).

\section{Domain Scores}

In general, the mean score in all domains was lower in RR/ MDR-TB than in drug-susceptible TB. Among RR/MDRTB patients' environmental domain was the most affected with a mean score of $40.63 \pm 10.72$, whereas among drugsusceptible TB cases, physical domain had the lowest mean score $(61.80 \pm 17.18)$ but much higher than the means score observed in RR/MDR-TB cases. Both environmental and physical domains were the two domains with a lower mean score in both groups with inverse ranking. In RR/MDR-TB and drug-susceptible TB patients, the psychological domain had the highest mean score of $48.28 \pm 20.83$ and $76.63 \pm 15.32$, respectively. The 
Table I Socio-Demographic Characteristics of Study Participants

\begin{tabular}{|c|c|c|c|}
\hline Variable & RR/MDR-TB $(n=46)$ & DS- TB $(n=46)$ & $\begin{array}{l}\text { Total } \\
\mathbf{N}(\%)\end{array}$ \\
\hline \multicolumn{4}{|l|}{ Age } \\
\hline $15-24$ & $6(13.0 \%)$ & $6(13.0 \%)$ & $12(13.0 \%)$ \\
\hline $25-34$ & $13(28.3 \%)$ & II (23.9\%) & $24(26.1 \%)$ \\
\hline $35-44$ & $13(28.3 \%)$ & $9(19.6 \%)$ & $22(23.9 \%)$ \\
\hline $45-54$ & $6(13.0 \%)$ & 7 (I5.2\%) & $13(14.1 \%)$ \\
\hline 55 and above & $8(17.4 \%)$ & $13(28.3 \%)$ & $21(22.8 \%)$ \\
\hline \multicolumn{4}{|l|}{ Sex/gender } \\
\hline Male & $33(71.8 \%)$ & $26(56.5 \%)$ & $59(64.1 \%)$ \\
\hline Female & $13(28.2 \%)$ & $20(43.5 \%)$ & $33(35.9 \%)$ \\
\hline \multicolumn{4}{|l|}{ Marital status } \\
\hline Single & $15(32.6 \%)$ & 18 (39\%) & $33(35.9 \%)$ \\
\hline Married/living as married & 27 (58.7\%) & 21 (47.5\%) & $48(52.2 \%)$ \\
\hline Separated/divorced/ & $4(8.7 \%)$ & $4(8.7 \%)$ & $8(8.7 \%)$ \\
\hline Widowed & $0(0 \%)$ & $3(6.5 \%)$ & $3(3.3 \%)$ \\
\hline \multicolumn{4}{|l|}{ Educational level (grades) } \\
\hline Illiterate & $12(26.1 \%)$ & 7 (I5.2\%) & $19(20.1 \%)$ \\
\hline Primary school (I-5) & $9(19.6 \%)$ & $2(4.3 \%)$ & II (II.9\%) \\
\hline Middle school (6-8) & 9 (19.6\%) & $13(28.2 \%)$ & $22(23.9 \%)$ \\
\hline High school (9-12) & $12(26.1 \%)$ & $20(43.5 \%)$ & $32(34.8 \%)$ \\
\hline Tertiary school (university/college) & $4(8.7 \%)$ & $4(8.7 \%)$ & $8(8.7 \%)$ \\
\hline
\end{tabular}

observed difference in mean score for all four domains was statistically significant $(\mathrm{p}<0.001)$ (Table 2.)

\section{Discussion}

This study revealed that TB has significantly impacted HRQOL of patients, with significant impairment in all domains. Several studies have previously documented the impairment in HRQOL in TB patients. ${ }^{7,23,27,28}$ In the current study, environmental and physical domains were the most affected domains in RR/MDR-TB and DS-TB patients, respectively. This is contrary to a finding which stated environmental domain as the least affected domain $^{29}$ but similar to the results of two studies conducted among DS-TB patients. ${ }^{30,31}$ Although TB diagnosis, management and related hospital admissions were provided free of charge to all TB patients, and RR/MDRTB patients were also offered monthly pocket money for the duration of the treatment, participants of the present study reported that they lacked money to cover their daily needs and felt unsafe in their everyday life. Additionally, they expressed "unpleasant" feelings with their transportation, leisure activities, and availability of necessary information in their daily lives. Studies have indicated that the financial impact of TB is more noticeable in RR/MDR-TB than DS-TB patients, mainly due to their inability to work and in some instances not being allowed to work. ${ }^{32}$ More

Table 2 Comparison of Health Related Quality of Life Among RR/MDR-TB and Drug Susceptible TB Patients, Transformed Score (0100)

\begin{tabular}{|l|c|c|c|}
\hline & RR/MDR-TB & DS- TB & \\
\hline Domain & Mean \pm Std. Deviation & Mean \pm Std. Deviation & $t$-test \\
Physical domain & $42.94 \pm 19.60$ & $61.80 \pm 17.18$ & $\mathrm{t}=4.9094 \mathrm{p}<0.001$ \\
Psychological domain & $48.28 \pm 20.83$ & $76.63 \pm 15.32$ & $\mathrm{t}=7.4342 \mathrm{P}<0.001$ \\
Social-relationship domain & $45.83 \pm 25.26$ & $67.39 \pm 19.71$ & $\mathrm{t}=4.5637 \mathrm{p}<0.001$ \\
Environment domain & $40.63 \pm 10.72$ & $66.17 \pm 15.26$ & $\mathrm{t}=4.5637 \mathrm{p}<0.001$ \\
\hline
\end{tabular}


importantly, TB remains a disease related to poverty as even the free TB care is unaffordable for the poor TBaffected households. ${ }^{33}$ Thus, financial support for RR/ MDR-TB patients could resolve the financial problems caused by the disease and associated psychological suffering. In addition, it could support affordability, access to care and encourage treatment compliance. ${ }^{6}$

In our setting, all RR/MDR-TB patients are admitted for 6-8 months in the national RR/MDR-TB referral hospital until culture-based sputum tests are converted to negative. Loss of productivity as a result of hospitalization in RR/MDR-TB patients was identified as one of the main contributors to indirect costs. ${ }^{34}$ Hence, this long admission time could have hindered patients' freedom of movement and work opportunities. In addition, during the continuation phase, RR/MDR-TB patients had to come to the hospital for drug refill and scheduled follow-ups. This mandates patients from remote areas to travel several kilometers to go to the hospital and exposes them to further expenses. ${ }^{11}$ On the contrary, DS-TB patients are managed in ambulatory care, which could have given them relatively better opportunities to work, exercise leisure activities, and movements than RR/MDR-TB patients. Furthermore, as treatment of DS-TB is offered at health facilities and community levels with the support of community health promoters, DS-TB patients could have fewer transportation challenges than RR/MDR-TB patients. It is noted that ambulatory care models for RR/ MDR-TB patients perform at least equivalent outcomes and may result in improved quality of life. ${ }^{14}$

In DS-TB patients, the physical domain was the top most affected domain, but the observed mean score of this domain was much lower in RR/MDR-TB patients. This finding is contrary to a result that mentioned physical domain as the least affected ${ }^{30}$ but consistent with another report with a much higher mean score than the current study. $^{34}$ Almost all RR/MDR-TB and some DS-TB patients testified that physical pain prevented them from doing desired activities; consequently, they depended on medical treatment for pain relief. Patients also complained about lack of energy and related dissatisfaction in their ability and capacity to perform daily living activities. Previous study has identified that physical ailments related to the disease reduce patients' capacity to work, which causes patients' feeling of incompetence and being a burden to their families. ${ }^{11}$ The treatment regimen for RR/MDR-TB includes consumption of multiple pills, accompanied by daily injection for the duration of the intensive phase (6-8 months), which could have potentially added and worsened the disease caused pain, as adverse effects of TB treatment including the harmful effects of injectable agents were noted as negative contributors to HRQOL among drug-resistant TB patients. ${ }^{9}$ On the other hand, DS-TB patients received therapy with all oral and fixed-dose combination for a relatively shorter duration. Furthermore, MDR-TB patients are anticipated to have higher rates of chronic disability due to the extended treatment duration. Even after being cured, MDR-TB patients suffered from persistent respiratory symptoms, residual radiological sequels, and impairments in lung functions. 36,37

The social relationship was also another domain in which the RR/MDR-TB patients had a lower mean score than DS-TB cases and was among the least affected domains in both study groups. Previous studies from India, Nigeria, and Saudi Arabia described the social relationship as the least affected domain. ${ }^{31,35,38}$ In the current study, participants were not satisfied with their relationships, support from friends, and sex life. Most of the time, MDR-TB patients become socially isolated and lose support networks due to hospitalization, stigma, and difficulties in maintaining family life. ${ }^{14}$ In some places, MDR-TB patients faced stigma from their own family, community, and health care workers. ${ }^{11}$ In contrast, DS-TB patients lived in their homes with their families and close to their social circle and networks during treatment. This could have given DS-TB patients a better platform to get family and friends support when compared to RR/MDR-TB patients, who were usually cared for by formal caregivers and detached from their family, friends, and social network, at least for the intensive phase. Hence, RR/MDRTB patients could have experienced reduced social interaction and may lose the sense of belongingness to their close societal circles.

Although the psychological domain was the least affected domain in both groups, RR/MDR-TB patients had a much lower mean score than DS-TB cases. This finding is consistent with a study that reported the psychological domain as the least affected one, ${ }^{39}$ but contrary to several studies that reported the opposite. ${ }^{29,35,40}$ Although more than half of our study participants reported that they had negative feelings and were not satisfied with themselves, they felt and viewed their life as meaningful and were able to accept their bodily appearance. The possible reason for the relatively higher mean score in this domain than in other domains cannot be directly speculated. But, 
study participants could have underestimated their true feelings about their psychological health as complaining about mental health issues is not a norm in Eritrean society. Society has taught them to be thankful for what they have and endure what they go through. It is noted elsewhere that when diagnosed with TB, some patients exhibit denial, reduced self-esteem, and present with negative feelings about the disease resulting in depression, anxiety, disappointment, and refusal to accept the diagnosis. ${ }^{11}$ Furthermore, psychosis has been reported as a side effect of some anti-TB drugs used to treat MDR-TB patients ${ }^{41}$ and occasionally leads to more significant psychological problems than the disease itself. ${ }^{6}$ Hence, RR/ MDR-TB patients could be exposed to a more significant psychological problem than DS-TB cases. Besides the clinical manifestations, social and individual behavioral aspects contribute to the high psychological distress in TB patients. $^{42}$

\section{Limitations of the Study}

The study's sample size was small despite the inclusion of all RR/MDR-TB patients on treatment, and the comparator group (DS-TB) was not matched to the RR/MDR-TB cases. However, as the study site was the only RR/MDRTB treatment center in Eritrea, the results for RR/MDRTB can be generalized. In addition, due to its crosssectional nature, this study did not provide data on the changes in health-related quality of life at this point in time. Hence, the findings of this study should be interpreted with caution given the above mentioned limitations.

\section{Conclusions}

This study revealed that the HRQOL was impaired in both groups, but RR/MDR-TB patients had a worse healthrelated quality of life with statistically significantly lower mean scores in all domains than DS-TB patients. Environmental and physical domains were the two most affected, whereas social and psychological domains were the two least affected domains in both DS-TB and RR/ MDR-TB patients.

The findings of this study emphasize the need for integrated psycho- and socioeconomic intervention to optimize the care and support patients need to reduce the negative impacts of the disease. The interventions could include financial, material, and psychosocial support. In addition, treatment should be decentralized as long hospital admission could be aggravating the negative impacts on TB patients' HRQOL. Furthermore, a prospective study with a higher sample size should be done to evaluate the HRQOL in different stages of treatment.

\section{Abbreviations}

DR-TB, drug-resistant TB; DS-TB, drug-susceptible TB; HIV, human immune deficiency virus; HRQOL, Healthrelated quality of life; QoL, quality of life; RR/MDR-TB, Rifampicin resistant/multidrug-resistant TB; RR, rifampicin resistant; SPSS, statistical package for social sciences; TB, tuberculosis; WHO, World Health Organization; WHOQOL-BREF, World Health Organization Quality of Life Bref.

\section{Data Sharing Statement}

The data set analysed in this study is available from the corresponding author on reasonable request.

\section{Ethics Approval and Consent to Participate}

The study was conducted in accordance with the Declaration of Helsinki. Ethical approval was obtained from the Health Research and Ethical committee of the Ministry of Health, Eritrea. Written informed consent was collected from all participants of the study and parental consent was obtained for cases under 18 years of age. Participation was voluntary and participants had the right to decline their participation at any time during the study period. Moreover, the data were collected anonymously and did not include any personal identifiers to ensure confidentiality.

\section{Consent for Publication}

Not applicable

\section{Acknowledgments}

The researchers would like to appreciate the cooperation and support of all staff and patients of Merhano Hospital.

\section{Author Contributions}

All authors made a significant contribution to the work reported, whether that is in the conception, study design, execution, acquisition of data, analysis and interpretation, or in all these areas; took part in drafting, revising or critically reviewing the article; gave final approval of the version to be published; have agreed on the journal to which the article has been submitted; and agree to be accountable for all aspects of the work. 


\section{Funding}

There is no funding to report.

\section{Disclosure}

All authors declare that they have no conflicts of interest for this work.

\section{References}

1. Global Tuberculosis Report 2020. Geneva:World Health Organization. 2020.

2. Ministry of Health 2020. National TB and Leprosy Control Program. Quarterly report. Asmara, Eritrea.

3. Mesfin AB, Araia ZZ, Beyene HN, et al. First molecular-based anti-TB drug resistance survey in Eritrea. INT $J$ of TUBERC LUNG DIS. 2021;25(1):43-51. doi:10.5588/ijtld.20.0558

4. World Health Organization. Consolidated Guidelines on DrugResistant Tuberculosis Treatment. Geneva: World Health Organization; 2019.

5. Seung KJ, Keshavjee S, Rich ML. Multidrug-resistant tuberculosis and extensively drug-resistant tuberculosis. Cold Spring Harb Perspect Med. 2015;5:a017863. doi:10.1101/cshperspect.a017863

6. Thomas BE, Shanmugam P, Malaisamy M, et al. Psycho-socioeconomic issues challenging multidrug resistant tuberculosis patients: a systematic review. PLoS One. 2016;11(1):e0147397. doi:10.1371/ journal.pone.0147397

7. Kastien-Hilka T, Rosenkranz B, Sinanovic E, Bennett B, Schwenkglenks M. Health-related quality of life in South African patients with pulmonary tuberculosis. PLoS One. 2017;12(4): e0174605. doi:10.1371/journal.pone.0174605

8. Ahmad N, Javaid A, Syed Sulaiman SA, et al. Effects of Multidrug Resistant Tuberculosis Treatment on Patients' Health Related Quality of Life: results from a Follow Up Study. PLoS One. 2016;11(7): e0159560. doi:10.1371/journal.pone.0159560

9. Sineke T, Evans D, Schnippe K, et al. The impact of adverse events on health related quality of life among patients receiving treatment for drug-resistant tuberculosis in Johannesburg, South Africa. Health Qual. Life Outcomes. 2019;17:94. doi:10.1186/s12955-019-1155-4

10. WHO. Programme on Mental Health. WHOQOL Measuring Quality of Life. Division of Mental Health and Prevention of Substance Abuse. World Health Organization; 1997.

11. Thiruvalluvan E, Thomas B, Suresh C, Sellappan S, Muniyandi M, Watson B. The psychosocial challenges facing multi drug resistance tuberculosis patients: a qualitative study. SAARC J Tuber Lung Dis HIV/AIDS. 2017;XIV(1).

12. Guo N, Marra F, Marra CA. Measuring health-related quality of life in tuberculosis: a systematic review. Health Qual. Life Outcomes 2009;7:14. doi:10.1186/1477-7525-7-14

13. Aggarwal AN. Quality of life with tuberculosis. J Clin Tuberc Other Mycobact Dis. 2019;17:100121. doi:10.1016/j.jctube.2019.100121

14. Brown J, Capocci S, Smith C, Morris S, Abubakar I, Lipman M. Health status and quality of life in tuberculosis. Inte $J$ Infect Dis. 2015;32:68-75. doi:10.1016/j.ijid.2014.12.045

15. Chamla D. The assessment of patients' health-related quality of life during tuberculosis treatment in Wuhan, China. Int J Tuberc Lung Dis. 2004;8:1100-1106.

16. Bauer M, Leavens A, Schwartzman K. A systematic review and meta-analysis of the impact of tuberculosis on health-related quality of life. Qual Life Res. 2013;22:2213-2235. doi:10.1007/s11136-0120329-x

17. Sumona Datta RH, Gilman RM, et al. Quality of life, tuberculosis and treatment outcome; a case-control and nested cohort study. Eur Respir J. 2020;56:1900495. doi:10.1183/13993003.00495-2019
18. Patel AE, Lalitha K, Dinesh Rajaram K, Radhika NSM. Quality of life among patients on MDR-TB treatment in a district tuberculosis centre of a metropolitan city. Int J Community Med Public Health. 2018;5(12):5355-5359. doi:10.18203/23946040. ijcmph20184816

19. Alene KA, Clements ACA, McBryde ES, et al. Mental health disorders, social stressors, and health-related quality of life in patients with multidrug-resistant tuberculosis: a systematic review and meta-analysis. $J$ Infect. 2018;77(5):357-367. doi:10.1016/j. jinf.2018.07.007

20. Louw JS, Mabaso M, Peltzer K. Change in health-related quality of life among pulmonary tuberculosis patients at primary health care settings in South Africa: a Prospective Cohort Study. PLoS One. 2016;11(5):e0151892. doi:10.1371/journal.pone.0151892

21. Ribeiro L, Sousa C, Sousa A, et al. Evaluation of hearing in patients with multi resistant tuberculosis. Acta Med Port. 2015;28(1):87-91. doi:10.20344/amp.5783

22. Conradie F, Mabiletsa T, Sefoka M, et al. Prevalence and incidence of symmetrical symptomatic peripheral neuropathy in patients with multidrug-resistant TB. S. Afr. Med. J. 2014;104(1):24-26. doi:10.7196/SAMJ.6455

23. Jaber AAS, Ibrahim B. Health-related quality of life of patients with multidrug-resistant tuberculosis in Yemen: prospective study. Health Qual. Life Outcomes. 2019;17:142. doi:10.1186/s12955-019-1211-0

24. Kastien-Hilka T, Abulfathi A, Rosenkranz B, Bennett B, Schwenkglenks M, Sinanovic E. Health-related quality of life and its association with medication adherence in active pulmonary tuberculosis-a systematic review of global literature with focus on South Africa. Health Qual. Life Outcomes. 2016;14:42. doi:10.1186/ s12955-016-0442-6

25. WHO. Programme on Mental Health. WHOQOL-BREF Introduction, Administration, Scoring and Generic Version of the Assessment. Field Trial Version. Geneva; 1996.

26. WHO. Programme on Mental Health. Division of Mental Health and Prevention of Substance abuse. WHOQOL manual. WHO/MNH/ MHP/98.4.Rev.1. English only. Distr.: General.

27. Jaber AAS, Khan AH, Syed Sulaiman SA, Ahmad N, Anaam MS. Evaluation of health related quality of life among tuberculosis patients in two cities in Yemen. PLoS One. 2016;11(6):e0156258. doi:10.1371/journal.pone. 0156258

28. Malik M, Nasir R, Hussain A. Health related quality of life among tb patients: question mark on performance of TB DOTS in Pakistan. J Trop Med. 2018;2018:1-7. doi:10.1155/2018/2538532

29. Ayub T, Nazir U, Sideeq K. Quality of life of multi drug resistant tuberculosis cases of Kashmir Valley. IJIRMS. 2017;02. doi:10.23958/ijirms/vol02-i08/03.

30. Sule AG, Odeigah LO, Alabi KM, et al. Quality of Life of Patients with Tuberculosis in a Nigerian Teaching Hospital. TJFMPC. 2014;8 (2):39-47. doi:10.5455/tjfmpc.46982

31. Adeyeye OO, Ogunleye OO, Coker A, et al. Factors influencing quality of life and predictors of low quality of life scores in patients on treatment for pulmonary tuberculosis: a cross sectional study. J Public Health Afr. 2014;5:366. doi:10.4081/jphia.2014.366

32. van den Hof S, Collins D, Hafidz F, Beyene D, Tursynbayeva A, Tiemersma E. The socioeconomic impact of multidrug resistant tuberculosis on patients: results from Ethiopia, Indonesia and Kazakhstan. BMC Infect Dis. 2016;16:470. doi:10.1186/s12879016-1802-x

33. Wingfield T, Tovar MA, Huff D, et al. Beyond pills and tests: addressing the social determinant of tuberculosis. Clin Med (Northfield Il). 2016;16(6):s79-s91. doi:10.7861/clinmedicine.166-s79

34. Ramma L, Cox H, Wilkinson L, et al. Patients' costs associated with seeking and accessing treatment for drug-resistant tuberculosis in South Africa. INT J TUBERC LUNG DIS. 2015;19(12):1513-1519. doi:10.5588/ijtld.15.0341 
35. Laxmeshwar C, Stewart AG, Dalal A, et al. Beyond 'cure' and 'treatment success': quality of life of patients with multidrugresistant tuberculosis. INT J TUBERC LUNG DIS. 2019;23 (1):73-81. doi:10.5588/ijtld.18.0149

36. Singla N, Singla R, Fernandes S, Behera D. Post treatment sequelae of multi-drug resistant tuberculosis patients. Ind $J$ Tuberc. 2009;56:206-212.

37. Nuwagira E, Stadelman A, Baluku JB, et al. Obstructive lung disease and quality of life after cure of multi-drug-resistant tuberculosis in Uganda: a cross-sectional study. Trop Med Health. 2020;48:34. doi:10.1186/s41182-020-00221-y

38. Al-Qahtani MF, El Mahalli AA, Al Dossary N, Al Muhaish A, Al Otaibi S, Al Baker F. Health-related quality of life of tuberculosis patients in the Eastern Province, Saudi Arabia. J. Taibah Univ. Medical Sci. 2014;9(4):311e317. doi:10.1016/j.jtumed.2014.04.005
39. Sartika I, Insani WN, Abdulah R. Assessment of Health Related Quality of Life among Tuberculosis Patients in a Public Primary Care Facility in Indonesia. J Glob Infect Dis. 2019;11(3):102-106. doi:10.4103/jgid.Jgid 13618

40. Sharma R, Yadav R, Sharma M, Saini V, Koushal V. Quality of life of Multi Drug Resistant Tuberculosis patients: a Study of North India. Acta Med Iran. 2014;52(6):448-453.

41. Yang TW, Park HO, Jang HN, et al. Side effects associated with the treatment of multidrug-resistant tuberculosis at a tuberculosis referral hospital in South Korea a retrospective study. Medicine. 2017;96:28. doi:10.1097/MD.0000000000007482

42. Vidyullatha P. Quality of life, psychological intervention and treatment outcome in Tuberculosis patients. The Indian scenario. Front. Pyschol. 2016;7:1664. doi:10.3389/fpsyg.2016.01664

\section{Publish your work in this journal}

Patient Related Outcome Measures is an international, peer-reviewed, open access journal focusing on treatment outcomes specifically relevant to patients. All aspects of patient care are addressed within the journal and practitioners from all disciplines are invited to submit their work as well as healthcare researchers and patient support groups.
The manuscript management system is completely online and includes a very quick and fair peer-review system. Visit http://www. dovepress.com/testimonials.php to read real quotes from published authors. 